\title{
Trabajo y gestación de alto riesgo por diabetes: dos circunstancias difíciles de conciliar
}

\author{
Janine Schirmer ${ }^{1}$
}

RESUMEN En 1991, la prevalencia de diabetes era de 7,6\% en mujeres de nueve capitales brasileñas. Esta enfermedad ya consta entre las principales causas de defunción en ese país y se está convirtiendo en un problema de salud pública cada vez más alarmante. A pesar de los avances en el tratamiento de la diabetes y una mejor atención obstétrica, la diabetes gestacional pone a la embarazada en alto riesgo de aborto espontáneo, cetoacidosis, hipoglucemia, hipertensión y otros trastornos graves. En este estudio se investigaron mediante historias de vida las relaciones entre salud, sexualidad y trabajo en cinco mujeres diabéticas embarazadas atendidas en el Ambulatorio de Atención Prenatal del Hospital San Pablo. Todas tenían diabetes mellitus no insulinodependiente, entre 26 y 43 años de edad y ganaban mensualmente de US\$150 a 375 por 8 horas de trabajo diario, con un descanso semanal. Dos tenían compañeros desempleados; todas realizaban las tareas domésticas del hogar durante el día de descanso. Las citas tomadas directamente de conversaciones con las cinco participantes revelan: la dificultad de cuidar los hijos y trabajar, el acoso sexual de que algunas son objeto, el trabajo como elemento de conflicto e insatisfacción, el mito de la felicidad natural de la maternidad, el control sexual que ejerce el hombre, la interferencia de las condiciones de trabajo y la actividad laboral con la gravidez, y la sensación de que están legalmente desamparadas debido a la falta de respeto de los empleadores por los derechos laborales y la protección de la maternidad que establece la Constitución Federal del Brasil. Se concluye que en las políticas de salud debe prestarse atención a mejorar la calidad de vida de las mujeres trabajadoras, especialmente si padecen diabetes y están embarazadas.

Este trabajo nació de una experiencia profesional como enfermera obstetra en un ambulatorio de asistencia prenatal a mujeres con gestación de alto riesgo, principalmente diabéticas. La motivación para llevar a cabo el trabajo fue una experiencia de nueve años de convivencia con estas pacientes y el conocimiento de primera mano de sus dificultades para conciliar el trabajo con el embarazo, muchas veces

\footnotetext{
1 Universidade Federal de São Paulo, Departamento de Enfermagem. Rua Napoleão de Barros, 754, CEP 04024-002 São Paulo, SP, Brasil. Correo electrónico (Internet): janine.denf@epm.br.
}

por la omisión y falta de respeto a sus derechos laborales. Es sabido que la tensión, la ansiedad y el miedo de perder el empleo dificultan el control metabólico de la diabetes y empeoran el estado de salud de la gestante y el pronóstico del embarazo.

Estas inquietudes me llevaron a investigar cómo perciben las mujeres sus derechos laborales durante el embarazo y la maternidad. Sin duda, esta percepción influye en la atención de enfermería, trascendiendo la asistencia clínico-obstétrica. La atención de enfermería es una relación en la cual no solo se comparten saberes sino también sentimientos. Por ello es fundamental un abordaje que incorpore la dimensión de la subjetividad, tanto en la relación de cuidar a otros como en la producción de conocimientos referentes a la salud y la enfermedad.

La epidemiología de la diabetes en el Brasil subraya el interés de este estudio. Según el Censo Nacional de Diabetes realizado por el Ministerio de Salud en 1991 en poblaciones urbanas de nueve capitales, la prevalencia de diabetes fue de $7,6 \%$ en mujeres y 7,5\% en varones. Las complicaciones degenerativas del síndrome diabético — principalmente la retinopatía, la nefropatía y 
la neuropatía- constituyen un serio problema de salud pública, ya que empeoran la calidad de vida del diabético, incapacitándolo a menudo y acortando su esperanza de vida.

La prevalencia de diabetes también ha aumentado considerablemente, con las consiguientes repercusiones socioeconómicas resultantes de las muertes prematuras y la incapacidad para el trabajo, así como los costos asociados con el control y el tratamiento de las complicaciones agudas y crónicodegenerativas de la enfermedad. En lo que respecta a la mortalidad por diabetes, esta ya consta entre las principales causas de defunción. En 1982, en Brasil, la tasa de mortalidad por diabetes fue de 9,4 por 100000 personas, lo que corresponde a $1,6 \%$ del total de defunciones. Con relación a los índices de morbilidad, que son indicadores más efectivos de las condiciones de salud y de la calidad de vida de una población, en diabéticos la enfermedad cardiovascular es tres veces más frecuente que en no diabéticos. La vasculopatía periférica aparece aproximadamente en uno de cada 10 diabéticos mientras que solo se da en 2,6\% de los no diabéticos. La diabetes es también la principal causa de ceguera adquirida y la segunda causa de nefropatía terminal, hallándose presente aproximadamente en 20 a $25 \%$ de estos cuadros clínicos.

Las mujeres con diagnóstico de diabetes previo o reconocido en la gestación son consideradas de alto riesgo. A pesar de que los avances en el cuidado del enfermo diabético y en la atención obstétrica y neonatal han promovido una mejora de los resultados perinatales y maternos, la diabetes gestacional representa la posibilidad de complicaciones graves. Las complicaciones maternas más frecuentes son el aborto espontáneo, la cetoacidosis, la hipoglucemia, la hipertensión, la pielonefritis y otras infecciones, el polihidramnios, el parto prematuro y el agravamiento de las complicaciones crónicas vasculares de la diabetes.

La mortalidad y la morbilidad de los recién nacidos de madres diabéticas están relacionadas con la alta incidencia de malformaciones congénitas, macrosomía fetal, trastornos metabóli- cos y síndrome de dificultad respiratoria del neonato.

Al estudiar la relación del trabajo con la gestación de alto riesgo se parte de una consideración diferente de la vivencia de la maternidad, como una circunstancia que se suma al desgaste de la mujer trabajadora, ya que consume energía, transforma su cuerpo, altera sus relaciones sociales $\mathrm{y}$, finalmente, requiere tiempo y dedicación para conseguir llevar la gestación a un buen término.

El conocimiento de la legislación que establece normas laborales es muy importante para que la mujer trabajadora pueda exigir sus derechos y saber cuáles son sus deberes como empleada. La Constitución Federal brasileña de 1988 asegura a los trabajadores urbanos y rurales derechos referentes a la maternidad y a la paternidad.

\section{METODOLOGÍA DEL ESTUDIO}

El presente estudio partió de un enfoque multidisciplinario de enfermería obstétrica, epidemiología y sociología de la salud. Se trata de una investigación de tipo cualitativo, un estudio de casos mediante historias de vida. Con la contribución de la antropología y la sociología este tipo de investigación permite profundizar en la comprensión del fenómeno y de los factores complejos que intervienen en la relación entre salud, sexualidad y trabajo. La postura metodológica adoptada hizo posible construir un espacio de confianza y reflexión entre la investigadora y las mujeres que participaron en la investigación. De esta manera hubo una identificación entre el objeto estudiado y los sujetos entrevistados.

La selección de los sujetos de la investigación se hizo mediante dos criterios: formar parte del grupo de embarazadas diabéticas atendidas en el Ambulatorio de Atención Prenatal del Hospital San Pablo y tener un vínculo laboral formal. Cinco mujeres cumplieron estos criterios en el período de estudio de 6 meses.

Las cinco embarazadas incluidas en la investigación tenían diabetes mellitus no insulinodependiente o tipo II, en la que los síntomas metabólicos son mínimos o nulos. Este tipo de pacientes no necesitan insulina para prevenir la cetonuria y no son propensos a la cetosis. No obstante, pueden necesitar insulina para corregir algunos síntomas como la hiperglucemia persistente.

Mediante entrevista en profundidad se exploró el perfil sociodemográfico, las formas de control en el trabajo, el acoso sexual en el lugar de trabajo, la salud, la sexualidad, la vida reproductiva y la relación entre trabajo y gestación de alto riesgo. Todas las entrevistas fueron grabadas en cinta magnetofónica. Se las trató de forma anónima y se procedió a análisis del discurso de las entrevistadas.

\section{Perfil sociodemográfico}

Las edades de las cinco embarazadas diabéticas estuvieron comprendidas entre 26 y 43 años. Dos eran casadas, hacía 15 y 23 años, respectivamente; las demás vivían en unión libre hacía 9 años como mínimo. Tres eran analfabetas y dos tenían primer grado incompleto. Cuatro trabajaban en restaurantes, servicios de limpieza o domicilios privados y la quinta como vendedora de ropa, en un centro comercial. Todas tenían registro de trabajo hacía más de un año y cumplían los requisitos legales que califican a un ciudadano como participante del mercado formal de trabajo. La legislación brasileña (Consolidación de Leyes del Trabajo) se refiere a esta categoría de trabajadora como "empleada ... que trabaja para una persona o empresa, por un período de tiempo, a cambio de un sueldo".

Los sueldos mensuales de las cinco mujeres estuvieron comprendidos entre un mínimo equivalente a US\$ 150 y un máximo equivalente a $\$ 375$ dólares, por 8 horas diarias de trabajo, con un descanso semanal. El marido de una de las casadas y el compañero de convivencia de otra de las embarazadas estaban desempleados.

El trabajo doméstico en su hogar formaba parte integral de la vida cotidiana de todas las mujeres investigadas. Todas ellas realizaban las tareas 
domésticas de su hogar, fundamentalmente durante el día de descanso, casi siempre con ayuda de la madre y las hijas mayores: ${ }^{2}$

Mi mamá vive conmigo y me ayuda, pero el trabajo pesado de la casa lo hago el día de descanso, el lunes (1).

Los quehaceres de la casa los hacen las gemelas de 17 años. Yo hago solamente la comida, cuando llego del trabajo (3).

Solamente una de las mujeres dejaba a su hija menor en la guardería, las demás dejaban a sus hijos con hermanos mayores, o solos:

Hago todo el trabajo de la casa. Tengo una hija de dos años, que se queda en la casa con la mayor de 17 (4).

Hago todo el trabajo de la casa. No confío en mi hija de 14 años que tiene ataques de epilepsia. A mi hija de dos años la dejaba en la guardería, pero ahora parece que perdí el derecho, por eso pago a una mujer para que la cuide, porque no puedo dejarla con la mayor (5).

Esta declaración muestra la dificultad de cuidar los hijos y trabajar, así como el desamparo, porque la noción de "derecho" es muy frágil.

\section{Control y acoso sexual en el lugar de trabajo}

Cuando se preguntó a las mujeres si se sentían controladas en el trabajo. solamente una afirmó no ser controlada, diciendo lo siguiente:

No, porque mi patrona es amiga y vecina mía. Ella abrió una cafetería, yo le ayudaba a hacer las meriendas. No me revisan a la salida del trabajo y tengo horario de almuerzo sin problema (3).

Tres de ellas indicaron formas concretas de control en el trabajo. Por ejemplo:

Sí, hay una persona supervisando, no deja estar conversando. Hay control de entrada y salida. Mi jefe es un hombre (4).

Controlan todo. Si una conversa mientras lava la vajilla le gritan: ja trabajar! Somos 11 mujeres en la cocina (5).

\footnotetext{
2 En las citas textuales de la entrevistas se indica la identidad de la mujer mediante un número del $1 \mathrm{al}$ 5 colocado al final de la cita, entre paréntesis.
}

Por otro lado, una de las mujeres no se sentía controlada. Por el contrario, era ella misma la que decía controlar a las compañeras. Se notaba por su forma de hablar que la responsabilidad que su jefe le había asignado le hacía valorar más su trabajo:

No tengo control, al contrario, soy yo quien controlo a la camarera y a la de la limpieza. Yo hago las compras de la casa. Si un día necesito por ejemplo salir más temprano, a cualquier cosa, no le pido permiso al patrón, que es chinchoso, sino a la patrona, usted sabe, que es la que paga el sueldo (3).

Las revisiones a la salida del turno, las restricciones para ir al servicio y la falta de respeto al horario de almuerzo son habituales en los medios laborales brasileños. Los relatos de las mujeres muestran la frecuencia con que se repiten estas formas de control.

Se revisan las bolsas a la salida. El horario de almuerzo es una hora, de 11 a 12 de la mañana. Yo me llevo la comida de casa (4).

La Constitución Federal brasileña asegura el tiempo para el descanso o alimentación: "La empleada que trabaja más de 6 horas continuas, tiene derecho a un intervalo mínimo de una hora para descanso o alimentación". Sin embargo, las entrevistas con las mujeres evidenciaron que a menudo no se respetaba esa legislación:

¿Horario para el almuerzo? No, yo
almuerzo detrás del mostrador, en el
intervalo entre una cliente y otra me
como la vianda, ya fría (1).
No hay revisión a la salida, pero las bol-
sas quedan en el servicio. Si una va dos
veces al servicio, empiezan los proble-
mas. Algunas veces yo no podía ir ni
siquiera una vez, ni cuando estaba con la
menstruación. No tenía descanso para el
almuerzo, comía a las cuatro de la tarde,
cuando nos daban un pequeño descanso
de 10 ó 15 minutos. Como soy diabética y
tomo insulina no puedo estar sin comer.
Cuando me contrataron ya sabían de mi
enfermedad (5).

El acoso sexual ya no es una forma de violencia socialmente ignorada, aunque a menudo se silencia en el medio laboral, casi siempre para no arriesgarse a la pérdida del empleo. Todas las mujeres habían sido testigo de acciones de acoso sexual, pero ninguna de ellas sabía si las víctimas habían denunciado los casos. Entre las mismas mujeres a menudo se juzga a la víctima como culpable de este tipo de violencia, según el dicho: "Si la mujer es seria no hay hombre sinvergüenza".

Acoso sexual lo vi en casi todos los lugares donde trabajé, pero la culpa es de la mujer, a muchas le gusta. Convidan para salir, dicen chistes... Yo creo que depende de la mujer. La mayoría sale con hombres. Si sucediera conmigo yo se lo diría a mi jefe (4).

No obstante, las entrevistadas afirmaron no sufrir acoso sexual en sus locales de trabajo:

La patrona viaja, yo me quedo en la casa sola con su marido y su hijo de 19 años, nunca ninguno me faltó el respeto (3).

\section{La salud}

Las entrevistadas perciben la enfermedad diabética como algo que irrumpe en sus vidas, como algo malo y grave:

Después de la diabetes mi salud se complicó (1).

Mi salud parece que no era tan buena como yo pensaba. Comenzaron a aparecer unos problemitas, la diabetes (2).

Mi salud no era buena ni mala. Solo después de esta diabetes mi salud dejó de ser buena (4).

Una de las mujeres relacionaba la pérdida de la "buena salud", con su venida a São Paulo y el trabajo:

Hasta 1988 estaba bien, luego engordé y me subió la tensión. Antes trabajaba en el campo, era delgada, tuve tres hijos sin problemas. Después de venir a São Paulo hace nueve años mi salud empeoró. Creo que debe haber sido por el trabajo, porque yo soy muy nerviosa: me gustan las cosas arregladas, no quiero que la patrona me esté llamando la atención. Yo pesaba 53 kilos, ahora peso 90 (3).

La maternidad tiene una fuerte repercusión en la posición que socialmente se adjudica a la mujer. La gravidez es socialmente considerada un "estado de gracia" y es difícil que se admitan manifestaciones en sentido contrario. Sin embargo, en los comen- 
tarios de las entrevistadas a menudo se cuestionó el mito de la felicidad natural de la maternidad, frente al trabajo como elemento de conflicto e insatisfacción. La gravidez es vista como causa de alteración de la salud, principalmente cuando el embarazo es de alto riesgo:

Lo único que es una complicación es el embarazo, y más con la diabetes que necesita control (1).

Los problemas de salud que tengo comenzaron después de que me hicieron la cesárea, no por la operación sino por la anestesia y ahora por el embarazo (4).

En cuanto al trabajo, las entrevistas revelan también una relación positiva con el mismo, quizá como forma de huir del confinamiento en el espacio doméstico:

Yo trabajo afuera hace ya mucho tiempo. Que trabaje es bueno porque me divierto, salgo de la rutina, yo me siento bien (5).

Al preguntarles si algún problema de salud limitaba su actividad cotidiana se mencionó el embarazo como razón de problemas físicos:

A veces me da un dolor en la espalda que me deja inmóvil. Una vez el patrón se levantó de madrugada y me puso una inyección para el dolor. Ahora con el embarazo estoy peor (3).

\section{Sexualidad}

En la percepción de la sexualidad de estas mujeres repercute intensamente lo relativo a la reproducción y la noción de fidelidad conyugal como obligación de la esposa. El control de la sexualidad y de la reproducción es ejercido por el marido, como rasgo cultural masculino y jerárquico de poder sobre la mujer. Algunas mujeres que dijeron tener compañero sexual permanente reafirmaron la fidelidad femenina y la confianza en la fidelidad masculina, sin dejar lugar para la duda en la relación o para la posibilidad de diálogo o de cualquier forma de negociación:

No. Por ahora no tiene otra mujer, pero ya la tuvo. En el otro embarazo nos separamos (1).

Mi marido es mi único compañero sexual y yo soy su única mujer (2).
Así como el embarazo fue considerado por las entrevistadas como un factor de deterioro de la salud física, también en la vida sexual induce efectos como depresión, ansiedad, indignación, conflicto y desajuste familiar:

Cada vez que estoy embarazada, nos separamos, quedamos mal. Cuando no estoy embarazada tenemos relaciones tres o cuatro veces por semana, pero en la gestación pasamos treinta días sin hacer nada. Él no me busca, necesito insistir, pero aun así es una cosa fría; y lloro y me desespero. Como nosotros ya nos separamos y él se buscó otra, ahora estoy más avivada; esto me preocupa todo el día. Cuando estaba de 8 meses hasta le di una zurra a la mujer que estaba con él y que vivía en mi mismo patio (1).

Mi vida sexual cambió después del embarazo, porque yo no quería más hijos. Fue un embarazo indeseado y si ahora él se siente rechazado, lo siento mucho, pero yo no quería. No me conformo con estar así. Cuando ahora él se me acerca me da asco. A veces cuando estoy en casa él viene y me dan esas náuseas. Él se acerca y yo le digo que se vaya, que no quiero nada con él (5).

Cuando el trabajo fue considerado causa de alteración de la vida sexual, esta se puso normalmente en relación con las condiciones desfavorables y la fatiga, no con la enfermedad:

$\mathrm{Ni}$ el embarazo ni el trabajo interfieren en nuestra vida sexual, porque nosotros solo nos vemos el fin de semana (3).

El trabajo interfiere en la vida sexual por causa del cansancio. Hay días que yo llego tan cansada que a duras penas doy de comer a los hijos y no quiero saber nada más, solo acostarme (4).

Todas las mujeres entrevistadas habían usado algún método anticonceptivo. No obstante, considerando que todas ellas tuvieron más de tres partos, muchos de ellos en la adolescencia, podemos afirmar que el uso de la anticoncepción fue bastante irregular y sin una adecuada asistencia profesional:

Yo tomaba píldoras y me embaracé con el DIU (3).

Yo usé inyección durante 8 años, luego píldoras y condón (4).

Tomé [nombre comercial de un anticonceptivo] durante siete años, antes de saber que tenía diabetes. Ahora estaba usando condón, pero me dio alergia en la vagina y la médica me recomendó no usarlo. Él se estaba cuidando. No conseguí cita en la planificación familiar porque no había cupo (5).

\section{Vida reproductiva}

Las cinco mujeres entrevistadas tuvieron su primera gestación entre los 15 y 21 años. Su trayectoria reproductiva estuvo marcada por un gran número de gestaciones, abortos y partos pretérmino, reducido período intergenésico, trastornos gravídicos como hipertensión, obesidad y diabetes, además de una atención sanitaria casi siempre inadecuada a la gestación, al parto y al puerperio.

La trayectoria reproductiva de la mujer $n^{0} 1$ está marcada por varias pérdidas gestacionales, probablemente resultado de la realización de actividades pesadas y extenuantes del trabajo doméstico y de problemas de la atención sanitaria y falta de información sobre los factores de riesgo que pueden desencadenar el parto prematuro.

En el primer embarazo hice control prenatal en una unidad básica de salud. No trabajaba fuera de casa pero lavaba ropa. Tenía que subir a una azotea para tenderla y un día que fui a subir tuve un sangrado y perdí mi hijo. El segundo embarazo eran gemelos. También hice control prenatal en el puesto y los exámenes los hacía en un hospital mejor. Pero yo no sabía que necesitaba cuidarme, trabajaba como cajera en un supermercado y con seis meses sentí dolores. No sabía que eran dolores de parto. Durante seis horas tuve cólico, comencé a sangrar y rompí aguas. Fui al hospital, nacieron dos niños de medio kilo, pero no sobrevivió ninguno.

En el tercer embarazo hice control prenatal aquí en el Hospital de San Pablo, en el grupo de aborto habitual. Con 32 semanas sentí contracciones así que me quedé hospitalizada durante tres días, recibiendo suero para evitar el parto. Me hacían monitoría fetal y en un examen vieron que el corazón del bebé iba muy despacio. Me hicieron cesárea de urgencia. En esa época la diabetes ya había aparecido, ya tomaba insulina. El bebé solo vivió dos días. El embarazo actual lo he deseado mucho pero las posibilidades de vida son mínimas por la gravedad de mi diabetes y mi hipertensión y la presencia de anticuerpos antifosfolípidos (1). 
La tercera gestante diabética tuvo primero una gestación única y luego otra gemelar. El parto fue en casa, en el campo, sin problemas:

Después me vine a vivir a São Paulo con este hombre y tuve un tercer embarazo que terminó en aborto provocado. No quería tener un hijo viviendo en la casa de la patrona. Mi marido buscó un empleo, nosotros no teníamos casa. La patrona pagó el aborto en una clínica, fue con anestesia pero sentí mucho dolor. Quedé en reposo dos días. Mi cabeza quedó buena porque yo estaba preocupada con mis hijos que estaban en Bahia, ya no podía tener más hijos (3).

Esta narración muestra el recurso del aborto como salida para defender a los hijos vivos mediante la conservación del empleo. Otra cuestión es la ambivalencia de la solidaridad de la patrona que paga el aborto garantizando la permanencia de la empleada en el trabajo y evitando nuevos gastos como consecuencia de la gestación.

En el cuarto embarazo tuve un aborto espontáneo y ahora estoy en este con diabetes que descubrieron hace tres años. El bebé apareció en la ecografía con labio leporino (3).

\section{Trabajo y gestación de alto riesgo}

Este grupo de embarazadas diabéticas manifestaron una ausencia de estímulo y reconocimiento por parte de los empleadores en lo referente a las necesidades de asistencia prenatal. La actividad laboral y las condiciones de trabajo interfirieron con la gestación, a lo cual contribuyó también el desconocimiento de las mujeres sobre sus derechos durante el embarazo. Desde el punto de vista ético estas cuestiones son indiscutiblemente una prioridad que debe ser enfrentada por los profesionales de salud:

El trabajo interfiere con mi embarazo. Para venir a control le dije a mi patrona que no podía ir a trabajar el miércoles. Donde yo trabajo hace mucho calor, yo lo paso mal, todo el día de pie y sin momento para ir al servicio. No recibo compensación adicional por trabajar en un ambiente insalubre, ni sé lo que es. En el Centro Comercial se pueden contar con los dedos de una mano los empleados que están registrados. Trabajan de 10 de la mañana a 10 de la noche, directo y no están registrados. Mi patrona me recuerda eso todos los días diciéndome que debería estar feliz porque estoy registrada (1).

La legislación del Brasil define el local de trabajo insalubre como "el lugar que puede ocasionar daño a la salud. Por ejemplo, lugares expuestos a calor, frío, polvo, ruidos, etc.". Los relatos de las entrevistadas aludieron a este tipo de condiciones.

Yo comencé a trabajar el último de los tres embarazos. Me resulta difícil trabajar durante el embarazo. El trabajo es pesado, hay que caminar mucho y al final del día mis pies están hinchados y tengo dolor en el bajo vientre. En este trabajo me dejan hacer control siempre que lleve un certificado y no falte, porque solo somos cuatro mujeres y si una falta descontrola el trabajo de las otras. Sé que mi embarazo es de alto riesgo, ya dije a mi jefe que puedo necesitar hospitalización, pero yo creo que no voy a tener problemas (4).

La protección a la maternidad fue ampliada en la Constitución del Brasil promulgada en 1988. Anteriormente la empleada tenía derecho a licencia de gestación o licencia de maternidad, de 90 días. La Constitución (art. 7, párr. XVIII) extendió esa licencia a 120 días, con pago del salario. Los siguientes comentarios de las gestantes diabéticas entrevistadas muestran cómo a menudo se incumple la legislación laboral que garantiza el derecho a licencia de maternidad:

Mi primer empleo con registro fue en mi tercer embarazo, donde comenzaron a descontarme los días que iba a control prenatal y a colectar exámenes. Ahí comencé a llevar declaración del médico y pararon de descontarme. La licencia de maternidad me la dieron, pero descontaron el pago correspondiente y el subsidio para transporte. Dijeron que yo no estaba allí para firmar el recibo y que no podían dar el dinero a mi marido. Cuando me reincorporé tuve que pleitear en la justicia para recibir el pago (5).

Según la Constitución y la Consolidación de Leyes del Trabajo (art. 476) la empleada no puede ser despedida desde la confirmación del embarazo hasta cinco meses después del parto y durante el período de licencia para tra- tamiento. Estos derechos a menudo no se respetan en la práctica:

En el último embarazo ya tuve problemas en el trabajo. Fui despedida porque quedé 5 días hospitalizada. Me dijeron que no podían quedarse con una mujer que vive enferma por un embarazo de alto riesgo. Por la diabetes no tenía problemas, pero por el embarazo sí (5).

Los derechos del trabajador, principalmente los relativos a la maternidad, deberían ser informados a las mujeres durante el embarazo y el puerperio por los profesionales de salud que prestan asistencia a la gestante. Las diabéticas de nuestra muestra solo obtuvieron información respecto a su licencia por maternidad en charlas con otras embarazadas, generalmente compañeras de trabajo:

Nadie me habló nunca sobre los derechos de la mujer embarazada, ni aquí ni en el prenatal. Cuando una pide un parte médico le dicen que no pueden dárselo, ni siquiera cuando una está hospitalizada. En la ley de ellos no vale la declaración o el certificado, solo vale el resumen de alta. Cuando una pide la declaración no le hacen caso (5).

La sexualidad puede ser a veces un medio para controlar y disciplinar el trabajo. El siguiente fragmento ilustra la afirmación anterior.

No sé qué es licencia por maternidad. Oí hablar de ella pero nadie me explicó qué es. Mi patrona me dijo que tengo derecho a esa licencia, pero primero me soltó unas indirectas para que abortara. Yo no quise. Mi marido tiene otros hijos, pero este es el primero conmigo (3).

Según la Constitución Federal (art. 7, párr. XIX), el hombre tiene derecho a 5 días de licencia por paternidad, contados a partir de la fecha en la que presenta al empleador la declaración de nacimiento. Los relatos de las mujeres mostraron por una parte el incumplimiento de la ley por las empresas y, por otra parte, la falta de información y educación de los trabajadores sobre sus derechos:

No sabía que el papá tiene derecho a licencia por paternidad (1).

Que el marido tiene derecho a licencia yo no lo sabía. A mi marido lo suspendieron ocho días del trabajo porque faltó el día que nació mi hijo (5). 


\section{Consideraciones finales}

Esta investigación apunta varias áreas temáticas a las que debe prestarse atención en las políticas de salud para conseguir mejorar la calidad de vida de las mujeres trabajadoras en general y de las diabéticas en particular.

Las mujeres diabéticas que quedan embarazadas se sienten doblemente culpadas. Por un lado, por tener una enfermedad que socialmente las estigmatiza como incapaces para ejercer su vida sexual y reproductiva; por otra parte, por haberse embarazado siendo diabéticas. Las exigencias del mundo del trabajo las penalizan duramente. Viven la gestación en permanente tensión por el miedo de perder el hijo y el empleo y ello puede llevar a desatender la propia salud, así como a la depresión, la baja autoestima y la desesperanza. En algunas de estas mujeres diabéticas la posibilidad de un nuevo embarazo es motivo de pánico. Por otra parte, la doble jornada de trabajo agrava los riesgos perinatales.

En la relación con los profesionales de salud y los empleadores a menudo se constatan problemas de discriminación y negación de los derechos de las mujeres. En lo que se refiere a los profesionales sanitarios esa situación muchas veces se debe a su falta de preparación para hacer frente a la complejidad de los problemas de estas mujeres, problemas que suelen rebasar en mucho el marco clínico de la enfermedad. Las empresas o los patrones privados se aprovechan tanto de la impunidad como de la falta de información de los trabajadores respecto a sus derechos.

Manuscrito recibido el 26 de junio de 1996 y aceptado para publicación el 10 de julio de 1996.

\section{BIBLIOGRAFÍA}

American Diabetes Association (ADA). Medical management of pregnancy complicated by diabetes. New York: ADA; 1993.

Brasil, Ministério da Saúde, Secretaria Nacional de Assistência à Saúde da Mulher, Divisão Nacional da Saúde Materno Infantil, SubPrograma de Assistência Integral à Saúde da Mulher. Gestação de alto risco. Brasília: 1991.

Brasil, Ministério da Saúde, Secretaria de Assistência à Saúde, Departamento de Assistência e Promoção à Saúde, Coordenação de Doenças Crônico-Degenerativas. Manual de diabetes, 2a ed. Brasília: 1993.

Bruschini MCA. Mulher, casa e família: cotidiano nas camadas médias paulistanas. Vértice: Fun- dação Carlos Chagas-Editora dos Tribunais; 1990.

Centro Feminista de Estudos e Assessoria. Guia dos direitos da mulher. Brasilia: 1994.

Instituto de Estudos da Religião (ISER). Programa Sofia: mulher, teologia e cidadania. III Seminário de Teologia e Direitos Reprodutivos. Petrópolis, RJ: ISER; 1994.

Minayo MCS. O desafio do conhecimento: pesquisa qualitativa em saúde. São Paulo/Rio de Janeiro: Hucitec-Abrasco; 1992

Oliveira ME, Storti R, Carneiro F. Gênero, saúde e trabalho: a dimensão oculta. São Paulo: Editora do Sindicato dos Bancários de São Paulo; 1996. (Distribuição Editora Brasiliense, São Paulo, SP, 1996.)
Romito P. Lavoro e salute in gravidanza: come la società si prende cura delle donne incite. Milano: Franco Angeli; 1990.

Schirmer J. Participação da enfermeira obstetra na equipe de assistência à gestante diabética (tesis de maestría). São Paulo: Escola Paulista de Medicina; 1989.

Schirmer J. Fatores de risco para o desenvolvimento de diabetes mellitus após diabetes diagnosticado na gravidez (tesis de doctorado). São Paulo: Universidade Federal de São Paulo; 1995.

World Health Organization. Diabetes mellitus: report of a WHO Study Group. Geneva: WHO; 1985. (Technical Reports Series 727)
ABSTRACT

Work and pregnancy at high risk from diabetes: two incompatible circumstances
In 1991 the prevalence of diabetes was 7.6\% in women in nine Brazilian state capitals. This disease now ranks among the leading causes of death in the country and is becoming an increasingly alarming public health problem. In spite of advances in the treatment of diabetes and improved obstetric care, gestational diabetes puts a pregnant woman at high risk of spontaneous abortion, ketoacidosis, hypoglycemia, hypertension, and other serious disorders. This study used life histories to investigate the relationship between health, sexuality, and work in five working women who were diabetic and pregnant and who received care at the Prenatal Care Outpatient Clinic of the San Pablo Hospital. All these women had non-insulin-dependent diabetes mellitus, were between 26 and 43 years old, and earned from US\$ 150 to 375 per month for working 8-hour days, with one day off each week. Two of the women's male partners were unemployed; all of the women performed household chores during their day off. Quotations taken directly from conversations with the five participants reveal: the difficulty of caring for children and working; the sexual harassment that some of them suffered; the conflict and dissatisfaction associated with work; the myth of the natural joy of motherhood; the sexual control exercised by males; the interference of working conditions and work activities with pregnancy; and the perception that they did not have legal protection owing to employers' lack of respect for workers' rights and for the maternity protection provisions of the Federal Constitution of Brazil. It is concluded that health policies should pay greater attention to improving the quality of life of working women, especially if they suffer from diabetes and are pregnant. 